\title{
SSRP1 silencing inhibits the proliferation and malignancy of human glioma cells via the MAPK signaling pathway
}

\author{
JIANMING LIAO $^{1 *}$, XIANG TAO $^{1 *}$, QIANSHAN DING ${ }^{2}$, JUNHUI LIU $^{1}$, XUE YANG $^{1}$, FAN-EN YUAN $^{1}$ \\ JI-AN YANG ${ }^{1}$, BAOHUI LIU ${ }^{1}$, GUO-AN XIANG ${ }^{3}$ and QIANXUE CHEN ${ }^{1}$ \\ Departments of ${ }^{1}$ Neurosurgery and ${ }^{2}$ Gastroenterology, Institute for Gastroenterology and Hepatology, \\ Renmin Hospital of Wuhan University, Wuhan, Hubei 430060; ${ }^{3}$ Department of General Surgery, \\ The Second People's Hospital of Guangdong Province, The Third Clinical Medicine School, \\ Southern Medical University, Guangzhou, Guangdong 510317, P.R. China
}

Received March 15, 2017; Accepted August 18, 2017

DOI: 10.3892/or.2017.5982

\begin{abstract}
Structure-specific recognition protein 1 (SSRP1) has been considered as a potential biomarker, since aberrant high expression of SSRP1 has been detected in numerous malignant tumors. However, the correlation between the expression level of SSRP1 and glioma remains unclear. The present study attempted to investigate the role of SSRP1 in the pathogenesis of glioma. In the present study, our data revealed that SSRP1 overexpression was detected in glioma tissues at both the mRNA and protein levels using quantitative real-time RT-PCR and immunohistochemical analysis. We also demonstrated that the upregulated expression of SSRP1 was correlated with the World Health Organization (WHO) grade of glioma. The knockdown of SSRP1 by siRNA not only resulted in the inhibition of cell proliferation, but also significantly inhibited glioma cell migration and invasion. Mechanistic analyses revealed that SSRP1 depletion suppressed the activity of the phosphorylation of the MAPK signaling pathway. In conclusion, the present study indicated that SSRP1 regulated the proliferation and metastasis of glioma cells via the MAPK signaling pathway.
\end{abstract}

\section{Introduction}

Cancer of the brain and the central nervous system is one of the 10 most common types of cancer in China, and its incidence rate has evidently increased from 2000 to 2011 (1). Glioma is the most common malignant primary brain tumor, and accounts for the vast majority of all intracranial tumors (2).

Correspondence to: Professor Qianxue Chen, Department of Neurosurgery, Renmin Hospital of Wuhan University, 238 Jie-Fang Road, Wuhan, Hubei 430060, P.R. China

E-mail: chenqx666@sohu.com

*Contributed equally

Key words: glioma, SSRP1, GEO data, proliferation, metastasis, MAPK pathway
At present, standard therapeutic options include maximal safe resection, followed by radiation therapy and adjuvant chemotherapy, which are not particularly effective (3). Despite the improvement of treatment modalities, the majority of patients with malignant glioma may have poor prognosis within 2 years of diagnosis (4).

Structure-specific recognition protein 1 (SSRP1) is a subunit of the facilitates chromatin transcription (FACT) complex, which attaches to the nucleosome and reassembles the nucleosome (5). SSRP1 was initially identified in 1991 as a high-mobility group protein 1 (HMG1)-related DNA binding protein, and the capacity of SSRP1 for biological functions can be attributed to its HMG domain (6). As an important histone chaperone, it plays a role as a transcription factor in the regulation of several targets to modulate cellular processes such as DNA replication, DNA damage repair, apoptosis and cell cycle regulation (7-11). It has been documented that the expression of SSRP1 was associated with the stage of cellular differentiation. High SSRP1 levels were observed in stem or less-differentiated cells, while low SSRP1 levels were observed in more differentiated cells (12). The high expression of SSRP1 was detected in multiple human types of cancer, including non-small-cell lung cancer, renal cell carcinoma, pancreatic ductal and colorectal adenocarcinoma, and hepatocellular carcinoma (13-15). In addition, it is a potential marker and target of aggressive types of cancer $(13,16,17)$.

The mitogen-activated protein kinase (MAPK) signaling pathway is a highly conserved module that regulates various cellular functions, including proliferation, differentiation and malignant transformation (18). The MAPK pathway has been reported to be activated in over $88 \%$ of gliomas (19). The suppression of MAPK signaling synergizes the cytotoxicity of receptor tyrosine kinase inhibitors in glioma tumor-initiating cells (20). Research on the function of SSRP1 has focused on directing nucleosome reorganization as the histone chaperone, while the expression and potential molecular mechanism of SSRP1 in glioma remains unknown.

In the present study, we evaluated the expression of SSRP1 in human patient samples and investigated its function in the progression of glioma. In addition, we explored the effect of SSRP1 silencing on glioma cell proliferation and invasion. 
The results revealed that SSRP1 may function as a regulator, and promote cell proliferation and invasion by enhancing the activity of the MAPK signaling pathway. Therefore, the present study revealed that SSRP1 played a role in the progression of glioma, and also provided valuable information for understanding the mechanism of glioma.

\section{Materials and methods}

Tissue samples. Human glioma tissues were obtained during surgery at the Department of Neurosurgery, Renmin Hospital of Wuhan University (Wuhan, China), from 2010 to 2016. These control non-glioma human brain tissues were collected from unmatched patients undergoing surgery for intracranial hypertension. A total of 83 paraffin-embedded tissue samples, which included 77 glioma tissues, and 6 normal brain (NB) tissues, were histopathologically diagnosed by two neuropathologists. For real-time quantitative reverse transcription-polymerase chain reaction (RT-PCR), 30 frozen (stored in liquid nitrogen) glioblastoma and $10 \mathrm{NB}$ tissues were evaluated. All tissues were classified according to the 2016 World Health Organization (WHO) classification of tumors of the central nervous system. Prior patient consent and approval from the Ethics Committee of Wuhan University were obtained for the use of these clinical materials. The authors assert that all experiments complied with the ethical standards of relevant national and institutional guidelines as well as the laws of the People's Republic of China. The detailed demographics of patients are presented in Table I.

Cells and cell culture. Human glioblastoma-derived cancer cell lines U118 and U251 were purchased from the Cell Bank Type Culture Collection of the Chinese Academy of Sciences (Shanghai, China). The American Type Culture Collection (ATCC; Manassas, VA, USA) suspects that U118 is a contaminated cell line which has similar cytogenetics and similar origin with glioblastoma cell line U138MG, since there is no doubt that U118 is a cell line derived from malignant gliomas, we thus used U118 as experimental cells. These cells were cultured in Dulbecco's modified Eagle's medium (DMEM) supplemented with $10 \%$ fetal bovine serum (FBS) (both from Gibco Grand Island, NY, USA), $100 \mathrm{U} / \mathrm{ml}$ of penicillin and $100 \mu \mathrm{g} / \mathrm{ml}$ of streptomycin (Sigma-Aldrich, St. Louis, MO, USA) at $37^{\circ} \mathrm{C}$ with $5 \% \mathrm{CO}_{2}$.

Gene expression profiles. SSRP1 expression datasets were obtained from the Gene Expression Omnibus (GEO) (http://www.ncbi.nlm.nih.gov/geo/). Three datasets were retrieved for the study: GSE50161 (13 NB, 15 pilocytic astrocytoma, 46 ependymoma, 22 medulloblastoma and 34 glioblastoma tissues were collected for microarray analysis), GSE4290 (23 NB and 157 glioma tissues were analyzed; glioma tissues comprised of 45 grade II, 31 grade III and 81 grade IV cases), and GSE3185 (10 astrocytomas and 10 glioblastoma tissues were analyzed). The expression values of SSRP1 were transformed to relative expression.

Immunohistochemical staining. The paraffin-embedded tissues were placed in xylene 3 times for $15 \mathrm{~min}$ at room temperature, hydrated in a series of $100,95,90,80,70$ and $60 \%$
Table I. Correlation between the clinicopathological characteristics and expression of SSRP1 protein in glioma.

\begin{tabular}{lllll}
\hline & \multicolumn{4}{c}{$\begin{array}{c}\text { SSRP1 } \\
\text { expression }\end{array}$} \\
\cline { 3 - 4 } Characteristics & $\begin{array}{c}\text { No. of } \\
\text { patients }\end{array}$ & High & Low & P-value \\
\hline Age (years) & & & & \\
$\geq 50$ & 42 & 28 & 14 & 0.223 \\
$<50$ & 35 & 21 & 14 & \\
Sex & & & & \\
Male & 39 & 27 & 12 & 0.535 \\
Female & 38 & 22 & 16 & \\
WHO grade & & & & \\
I+II & 11 & 3 & 8 & 0.009 \\
III+IV & 66 & 46 & 20 & \\
\hline
\end{tabular}

SSRP1, structure-specific recognition protein 1; WHO, World Health Organization

ethanol solutions, and washed in phosphate-buffered saline (PBS). The sections were treated with $3 \% \mathrm{H}_{2} \mathrm{O}_{2}$ and subjected to antigen retrieval by citrate buffer $(10 \mathrm{mmol} / \mathrm{l}, \mathrm{pH}$ 6.0) for $15 \mathrm{~min}$. Then, the sections were incubated with primary rabbit anti-SSRP1 polyclonal antibody (1:200; GeneTex, Inc., Irvine, CA,. USA) overnight at $4^{\circ} \mathrm{C}$. After washing with PBS, the slides were incubated with poly-HRP goat anti-rabbit antibody (Maixin Bio, Fujian, China) for $30 \mathrm{~min}$, and incubated with diaminobenzidine for $5 \mathrm{~min}$. Next, the sections were counterstained with hematoxylin, dehydrated in ethanol and coverslips were placed on the slides. Images were captured using an Olympus BX40 microscope and the CC-12 Soft Imaging System (Olympus, Tokyo, Japan).

Immunohistochemical evaluation. SSRP1-positive cells displayed brownish yellow granules on the cytoplasm. These results were evaluated through immunohistochemical scores (IHC scores), according to the intensity of the staining and the percentage of immunoreactive cells. The estimate of the staining intensity was scored as follows: 0 , no staining; 1 , weak staining; 2, moderate staining; and 3, strong staining. The percentage was rated on a scale of $0-4$, as follows: $0,<5 \%$; $1,5-25 \% ; 2,26-50 \% ; 3,51-75 \%$; and 4, 76-100\%. The IHC score was obtained by multiplying the percentage and intensity score. These IHC scores ranged from 0 to 12 , in which scores $0-4$ were considered as low expression, while scores 5-12 were considered as high expression. These scores were independently determined by two independent senior pathologists.

Western blot analysis. Cells were lysed in ice-cold RIPA

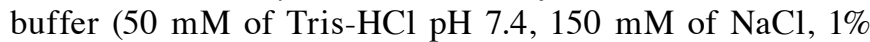
Triton X-100, $0.25 \%$ deoxycholate, $1.5 \mathrm{mM}$ of $\mathrm{MgCl}_{2}, 1 \mathrm{mM}$ of EGTA, $1 \mathrm{mM}$ of phenylmethylsulfonyl fluoride, $10 \mathrm{mM}$ of $\mathrm{ZnAF}, 10 \mathrm{mM}$ of pervanadate, $10 \mu \mathrm{g} / \mathrm{ml}$ of leupeptin and $10 \mu \mathrm{g} / \mathrm{ml}$ of aprotinin) and incubated for $30 \mathrm{~min}$. The protein concentration was determined using the BCA method. The cell lysate was heated at $100^{\circ} \mathrm{C}$ for $10 \mathrm{~min}$ after being mixed 
with sample loading buffer. The protein samples were equally loaded on $10 \%$ SDS-PAGE, and transferred onto nitrocellulose membranes. After being blocked with 5\% non-fat milk in Trisbuffer, the membrane strips were incubated with a primary antibody overnight at $4^{\circ} \mathrm{C}$, followed by Alex Fluor 680/790labeled goat anti-rabbit or goat anti-mouse IgG ( $\mathrm{Li}-\mathrm{COR}$ Biosciences, Lincoln, NE, USA). The strips were visualized using the LI-COR Odyssey Infrared Imaging System (Li-COR Biosciences).

The primary antibodies were as follows: p38, phospho-p38, ERK, phospho-ERK, JNK and phospho-JNK (1:1,000; Cell Signaling Technology, Danvers, MA, USA); MMP2, VEGF, EGFR, cyclin D and E (1:1,000; Abcam, Cambridge, MA, USA); p65, Bcl2, Snail, c-MYC, SSRP1 and GAPDH (1:1,000; GeneTex, Inc.).

RNA isolation and RT-PCR. Total RNA was isolated from U118 and U251 cell lines, and extracted from NB and glioma tissues with TRIzol reagents (Invitrogen, Carlsbad, CA, USA). Then, cDNA was prepared from $2 \mu \mathrm{g}$ of total RNA using the PrimeScript RT reagent kit with gDNA Eraser (Takara, Tokyo, Japan) according to the manufacturer's instructions. The primers were as follows: SSRP1 forward, 5'-GGATTGAAAG AGGGCATGAA-3' and reverse, 5'-AGAGGCGTTGCTGTC AAACT-3'; and GAPDH forward, 5'-ACAACTTTGGTATCG TGGAAGG-3' and reverse, 5'-GCCATCACGCCACAGT TTC-3'. RT-PCR was performed to quantify the mRNA expression using the SYBR-Green PCR Master Mix (Takara). GAPDH was used for SSRP1 normalization.

Transient transfection with siRNAs. Small interfering RNAs (siRNAs)weredesignedand synthesizedbySuzhouGenePharma, Inc. (Shanghai, China). Two siRNAs targeting the SSRP1 gene were designed and purchased, and the most effective siRNAs were identified using both RT-PCR and western blotting. The sequences of the siRNAs against SSRP1 were as follows: siRNA1 sense, 5'-GCCAUGUCUACAAGUAUGATT-3' and antisense, 5'-UCAUACUUGUAGACAUGGCTT-3'; siRNA2 sense, 5'-CCCAGAAUGGUGUUGUCAAATT-3' and antisense, 5'-UUUGACAACACAUUCUGGGTT-3'. The sequence of the negative control siRNA was as follows: sense, 5'-UUCUCCGAACGUGUCACGUTT-3' and antisense, 5'-ACGUGACACGUUCGGAGAATT-3'. The siRNA sequences were derived from another research group at our laboratory, and the specificity had been verified (14). Glioma cells were plated onto a 6 - or a 96 -well plate at $40-50 \%$ confluency. After $24 \mathrm{~h}$, siRNA transfections were conducted using HyperFect (Qiagen, Hilden, Germany), according to the manufacturer's protocol. Cells were harvested after 48-72 h. All siRNAs were used at a final concentration of $20 \mathrm{nM}$.

Cell proliferation assay. Cell proliferation was analyzed using Cell Counting Kit-8 (CCK-8; Dojindo, Tokyo, Japan). Cells were seeded in 96-well plates on day 0 , and the cell growth was assessed at days 1,2 and 3 after culturing, according to the manufacturer's instructions. On average, 5 replicates for each time-point were statistically analyzed.

5-Ethynyl-2'-deoxyuridine (EdU) incorporation assay. Cells were seeded into 96-well plates containing complete medium, and were allowed to attach overnight. Then, the cell growth was determined using the CellLight Edu imaging detecting kit (RiboBio, Guangzhou, China) according to the manufacturer's protocol. In brief, the cells were cultured in medium with $10 \mu \mathrm{M}$ of EdU for $24 \mathrm{~h}$, and fixed with $4 \%$ paraformaldehyde for $20 \mathrm{~min}$. Subsequently, the cells were incubated in an Apollo reaction cocktail for $30 \mathrm{~min}$, and the cell nuclei were stained with $5 \mu \mathrm{g} / \mathrm{ml}$ of Hoechst 33342 for $30 \mathrm{~min}$. The cells were visualized under a fluorescence microscope (Olympus BX51; Olympus, Tokyo, Japan). Experiments were performed in triplicate.

Cell cycle analysis. Cells were harvested with $0.25 \%$ trypsin and washed twice with ice-cold PBS. After centrifugation, the cells were suspended with $75 \%$ methanol overnight at $-20^{\circ} \mathrm{C}$. Then, the cells were washed twice with PBS, incubated in PBS with $1 \mathrm{mg} / \mathrm{ml}$ of RNAase at $37^{\circ} \mathrm{C}$, and stained with $50 \mathrm{mg} / \mathrm{ml}$ of propidium iodide (PI) in PBS. For each experiment, $2.5 \times 10^{4}$ cells were analyzed using BD FACSAria (BD Biosciences, Franklin Lakes, NJ, USA). Six replicates for each time-point were statistically analyzed.

Apoptosis analysis. Cells in each group were plated in 6-well plates, and harvested at $48 \mathrm{~h}$ after transient transfection with siRNA. Then, the cells were stained with ApoScreen Annexin V and PI, according to the manufacturer's instructions (BD Biosciences). For each experiment, $2.5 \times 10^{4}$ cells were analyzed using BD FACSAria (BD Biosciences). Six replicates for each time-point were statistically analyzed.

Cell migration and invasion assays. The cell migration assay was conducted using $8-\mu \mathrm{m}$ pore size Transwell chambers (Corning, Corning, NY, USA). Cells in each group were suspended in serum-free DMEM. Then, $5 \times 10^{4}$ cells in $100 \mu \mathrm{l}$ of DMEM were plated into the upper chamber, and $600 \mu \mathrm{l}$ of DMEM containing $10 \% \mathrm{FBS}$ was added to the lower chamber. These chambers were cultured at $37^{\circ} \mathrm{C}$ with $5 \% \mathrm{CO}_{2}$ for $24 \mathrm{~h}$. The cells in the upper chamber of the insert were collected using a cotton swab. Cells that had adhered to the lower surface were fixed with $4 \%$ paraformaldehyde, stained with $0.1 \%$ crystal violet and counted under a microscope (Olympus BX51). The invasion assay was the same, except that the Transwell chambers were precoated with Matrigel (R\&D Systems, Minneapolis, MN, USA), and the cells were cultured for $36 \mathrm{~h}$.

Statistical analysis. All statistical analyses are presented as the average of at least triplicate samples and as the mean \pm standard deviation. SPSS 19.0 (SPSS, Inc., Chicago, IL, USA) and GraphPad Prism 6.0 software (GraphPad Software, Inc., La Jolla, CA, USA) were used for statistical analysis. A $\mathrm{P}$-value $<0.05$ was considered statistically significant.

\section{Results}

SSRP1 mRNA and protein are overexpressed in glioma tissues. In order to observe the mRNA expression level of SSRP1 in glioma, 3 independent GEO datasets (GSE50161, GSE4290 and GSE3185) were analyzed. As shown in Fig. 1A, the expression of SSRP1 was upregulated in tumor tissues, compared to NB 
A
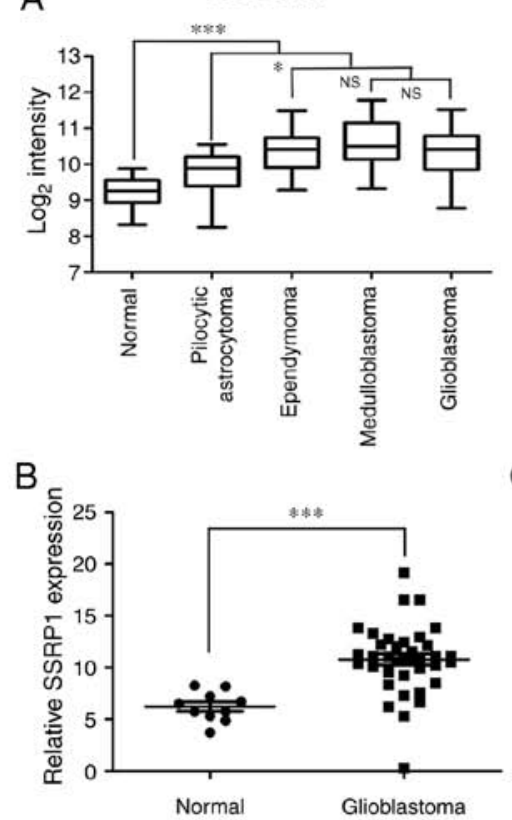
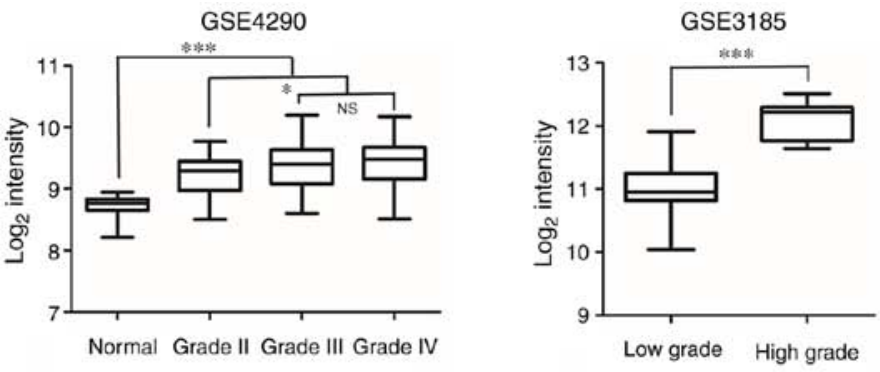

C

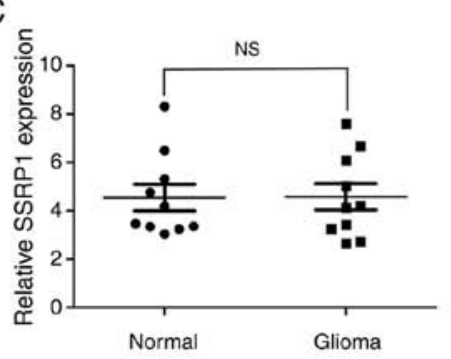

D

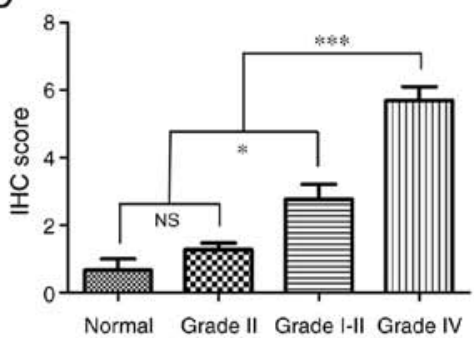

E

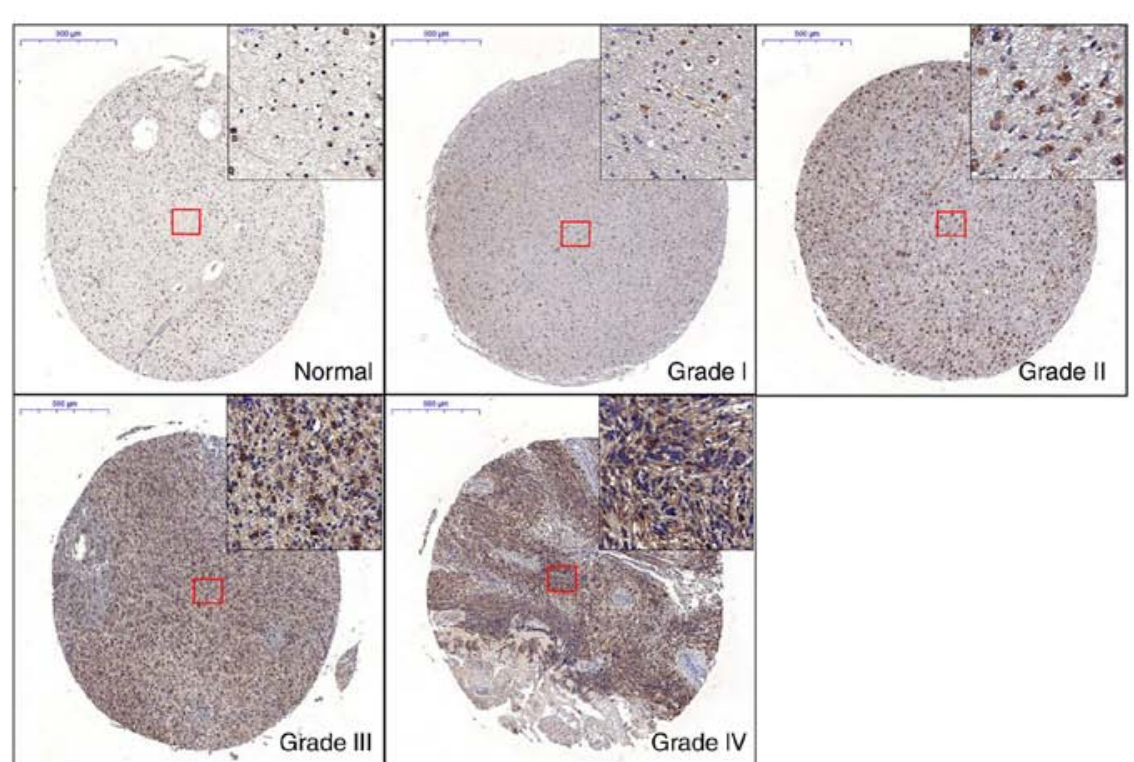

Figure 1. Expression of structure-specific recognition protein 1 (SSRP1) in human glioma tissues. (A) The mRNA expression level of SSRP1 in 3 datasets from the GEO repository (GSE50161, GSE4290 and GSE3185). The expression of the SSRP1 gene was higher in glioma tissues than in normal brain (NB) tissues. (B) SSRP1 mRNA expression was analyzed in 30 glioblastoma and 10 NB tissues by RT-PCR. (C) The fold change of SSRP1 expression in blood samples of 10 patients with glioblastoma and 10 normal control subjects. (D) Graphical representation of the IHC scores of the SSRP1 protein in 83 paraffin-embedded tissue samples. (E) The protein expression of SSRP1 in 83 paraffin-embedded tissue samples was assessed by immunohistochemical (IHC) staining. Representative images of the IHC staining for the SSRP1 protein is shown (magnification, $\mathrm{x} 100$ ), the top right corner are the microscopic images captured at a magnification of $\mathrm{x} 400$. Each bar is presented as the mean \pm standard deviation $(\mathrm{SD}) ;{ }^{*} \mathrm{P}<0.05,{ }^{* * *} \mathrm{P}<0.001$. NS, no significance.

tissues; and the expression levels of SSRP1 were significantly higher in the glioblastoma multiform group, compared with the low-grade glioma group. The mRNA expression of SSRP1 in $10 \mathrm{NB}$ and 30 glioblastoma tissues were examined by RT-PCR. Compared with NB tissues, glioblastoma tissues exhibited higher SSRP1 mRNA expression levels $(\mathrm{P}<0.001$; Fig. 1B). Next, we attempted to elucidate the clinicopathological significance of the mRNA expression of SSRP1 in the peripheral blood of glioma patients, and found that the difference between glioma patients and normal controls was not statistically significant ( $\mathrm{P}=0.97$; Fig. 1C). In addition, the protein expression levels of SSRP1 were assessed in an independent cohort of 83 cases, which included 6 NB cases, 3 grade I, 8 grade II, 22 grade III and 44 grade IV cases, using immunohistochemical staining. The staining density of SSRP1 in the high-grade group exhibited stronger coloring and broader distribution, compared to that in the low-grade group (Fig. 1E). As shown in Table I, among the 11 cases of glioma tissues at grades I-II, $3(27.3 \%)$ cases revealed positive staining, while 11 out of $22(50.0 \%)$ cases of glioma tissues at grade III and 35 out of 44 (79.5\%) cases of glioma tissues at grade IV were detected with positive staining of SSRP1 via immunohistochemical analysis. Quantitative analysis also revealed that the average score of SSRP1 staining increased from histologic grade I to grade IV ( $\mathrm{P}<0.05$; Fig. 1D). 


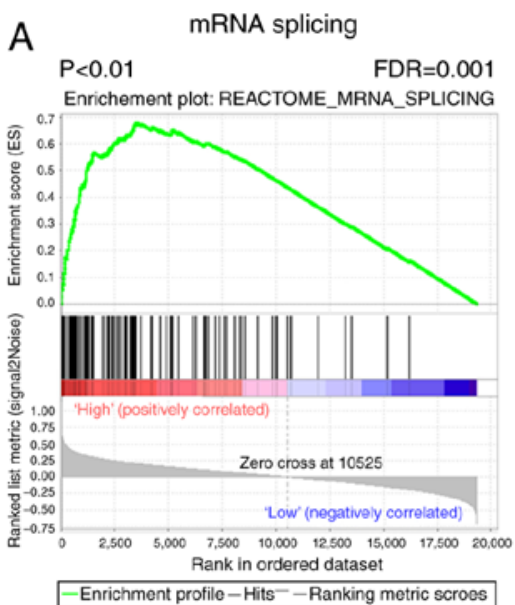

Enrichment Score $=0.677$

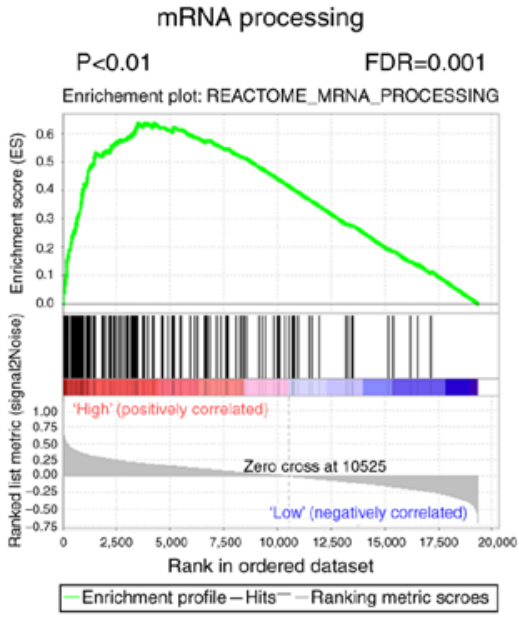

Enrichment Score $=0.637$

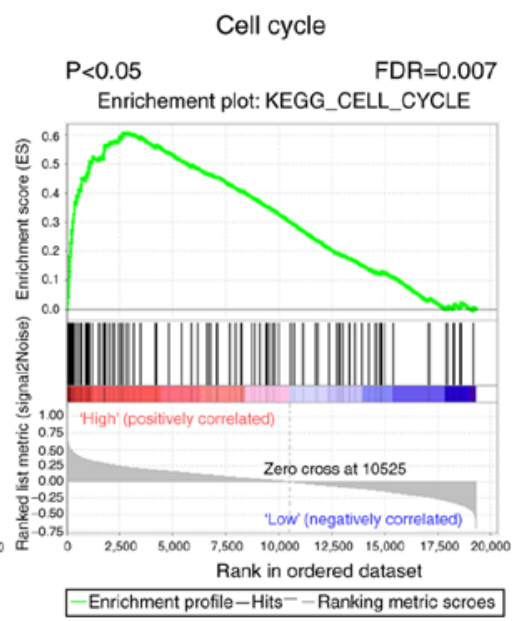

Enrichment Score $=0.607$
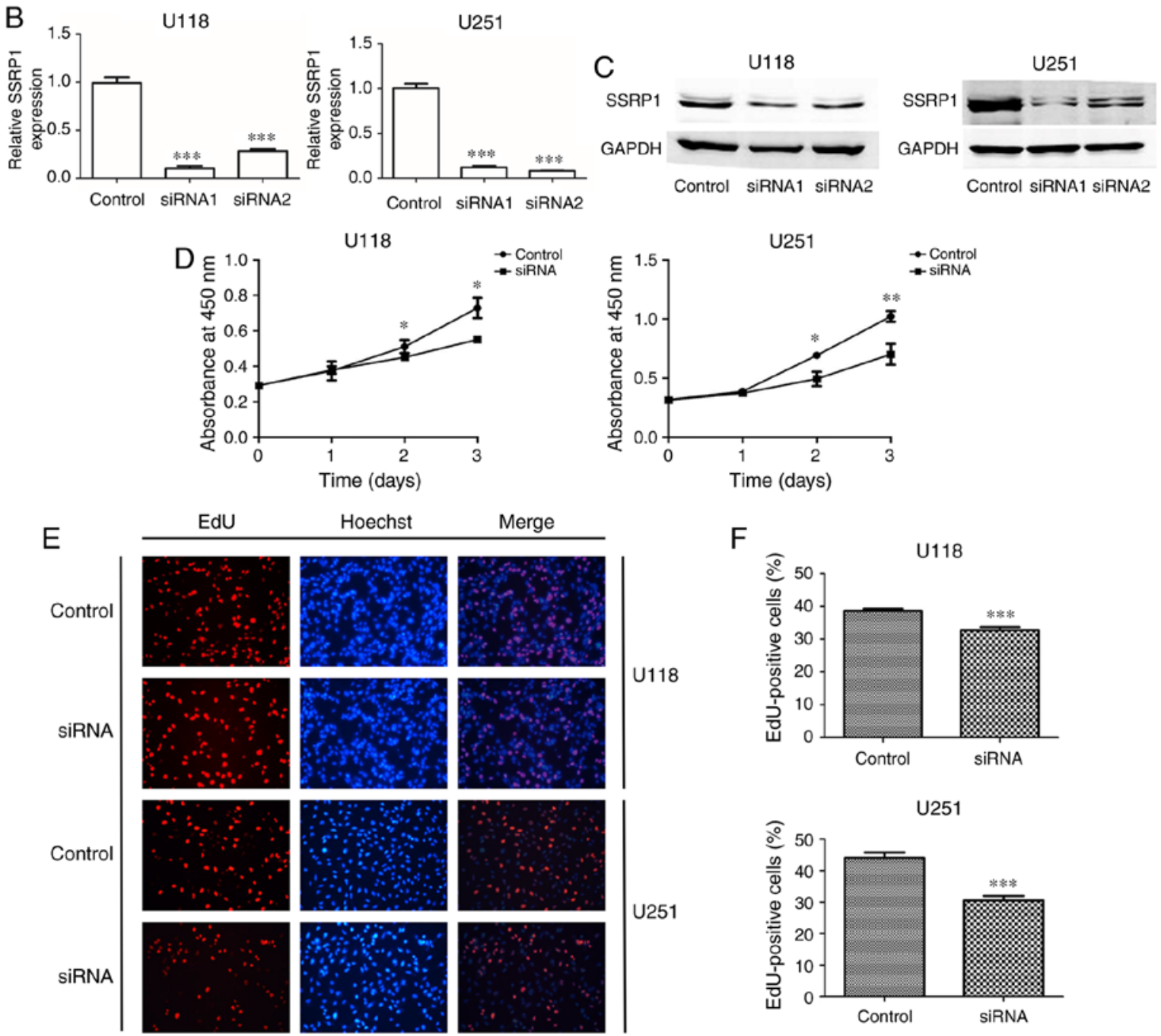

U118

F
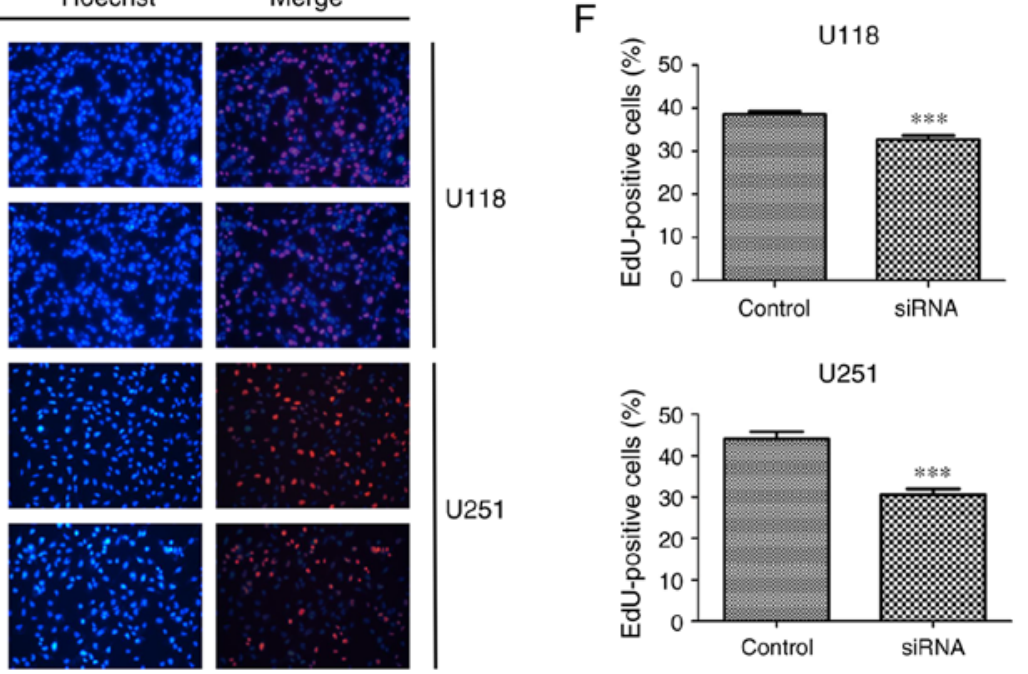

Figure 2. Downregulation of structure-specific recognition protein 1 (SSRP1) inhibits the proliferation in glioma cells. (A) The gene set enrichment analysis (GSEA) of GSE50161 revealed that SSRP1 may be involved in the progression of mRNA splicing, mRNA processing and the cell cycle. (B and C) The knockdown of SSRP1 by siRNA in U118 and U251 cells at both the mRNA and protein levels is shown. (D) Silencing of SSRP1 inhibited cell growth from day two as determined by CCK-8 assay in U118 and U251 cells. (E and F) EdU incorporation assay was performed to assess the DNA replication of U118 and U251 cells. Nuclei were stained with Hoechst (blue), and the proliferative cells were dyed red with EdU. A graphical representation of the percentage of EdU-positive cells is presented; ${ }^{*} \mathrm{P}<0.05,{ }^{* *} \mathrm{P}<0.01,{ }^{* * *} \mathrm{P}<0.001$. Data is presented as the mean \pm standard deviation (SD).

However, it was found that there was no significant association between the expression of SSRP1 and the age and sex of these patients (Table I).
Downregulated SSRPI expression suppresses cell proliferation in vitro. Gene set enrichment analysis (GSEA) was performed to identify biological processes potentially modulated by 


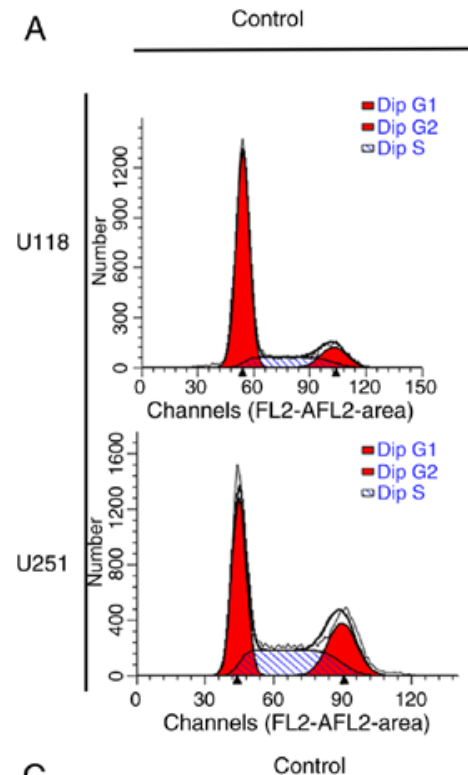

C
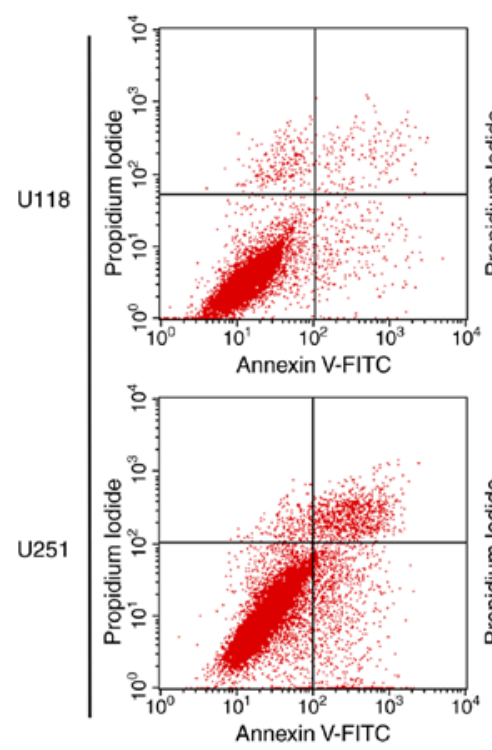
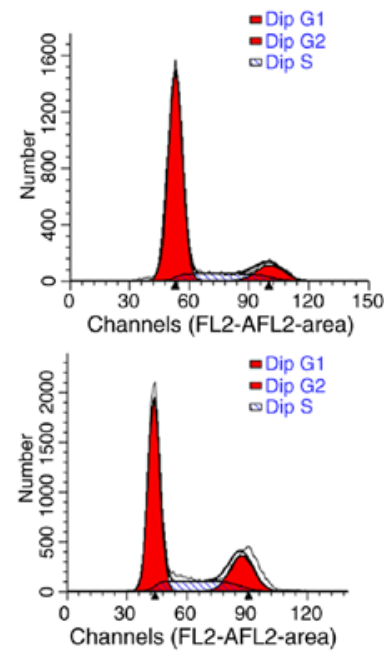

SIRNA
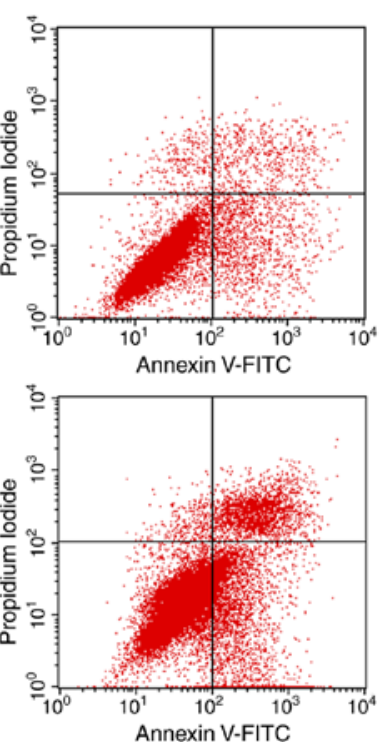

B
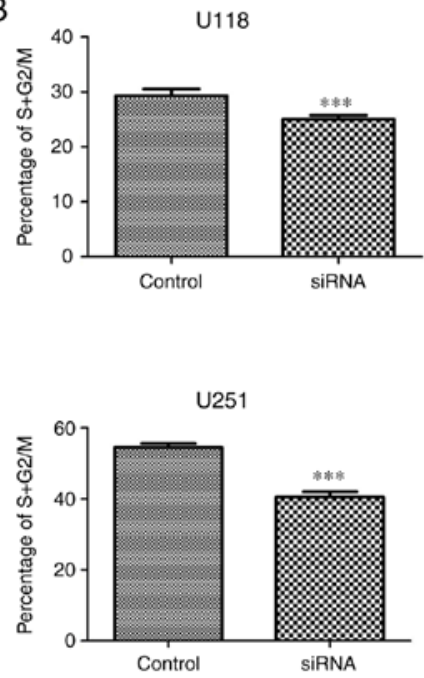

$\mathrm{D}$

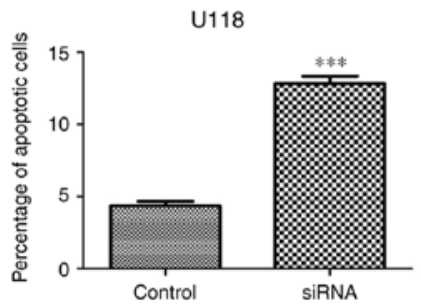

U251

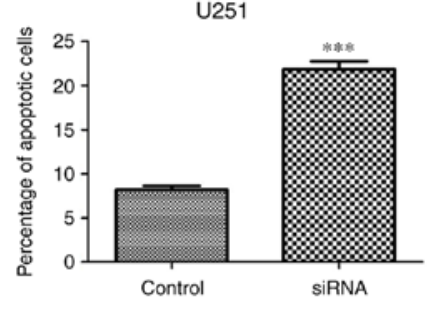

Figure 3. Effect of structure-specific recognition protein 1 (SSRP1) on the cell cycle and apoptosis. (A and B) Silencing of SSRP1 arrested the cell cycle in both U118 and U251 cells as dtermined by flow cytometric analysis. A graphical representation of the mean \pm standard deviation (SD) data revealed the percentage of cells in the $\mathrm{S}+\mathrm{G} 2 / \mathrm{M}$ phase. (C) The apoptosis rate of cells was assessed with Annexin V-FITC/propidium iodide by flow cytometry. (D) The quantitative analysis of apoptotic rates; ${ }^{* * *} \mathrm{P}<0.001$. Data are presented as the mean $\pm \mathrm{SD}$.

SSRP1. As shown in Fig. 2A, the GSEA results from gene profiling data (GSE50161) demonstrated that a mass of gene sets were enriched by SSRP1, and the enriched expression of gene sets included mRNA splicing, mRNA processing and the cell cycle (Fig. 2A).

A plasmid pcDNA3.1-SSRP1 was constructed to increase the expression of SSRP1 in glioma cells. U251 cells transfected with pcDNA3.1-SSRP1 plasmid (U251-SSRP1) has a higher mRNA expression level, while the protein level was only 1.20fold. The average percentage of EdU-positive U251-SSRP1 cells was similar with that in the U251 cells transfected with empty vector (38.30 and 39.51\%; $\mathrm{P}=0.502$ ). Flow cytometric analysis revealed that the average percentage of U251-SSRP1 cells in the $\mathrm{S}+\mathrm{G} 2 / \mathrm{M}$ phase was $52.17 \%$, which had no significant differences with the control group $(\mathrm{P}=0.102)$. Overexpression of SSRP1 could not exert its biological effects in U251, and the reason may be attributed to the high expression of exogenous expression of SSRP1. In addition, further increased expression of SSRP1 was at variance with objective reality. Thus, we did not perform a functional study of the overexpression SSRP1.

siRNA was used to specifically knockdown the expression of SSRP1 in U118 and U251 cell lines, which were established from high-grade glioma tumors. The efficient knockdown of SSRP1 expression was assessed through both RT-PCR and western blotting, and siRNA1 revealed more interference efficiency $(\mathrm{P}<0.001$; Fig. $2 \mathrm{~B}$ and $\mathrm{C})$. Therefore, siRNA1 was selected to knockdown the expression of SSRP1 in U118 and $\mathrm{U} 251$.

Subsequently, we verified the effect of the downregulated expression of SSRP1 in glioma cell growth in vitro. A CCK-8 assay was used to evaluate U118 and U251 cell growth at 24,48 and $72 \mathrm{~h}$ after siRNA transfection. The results revealed that the cell growth was significantly decreased in cells transfected with si-SSRP1 compared with the cells transfected 

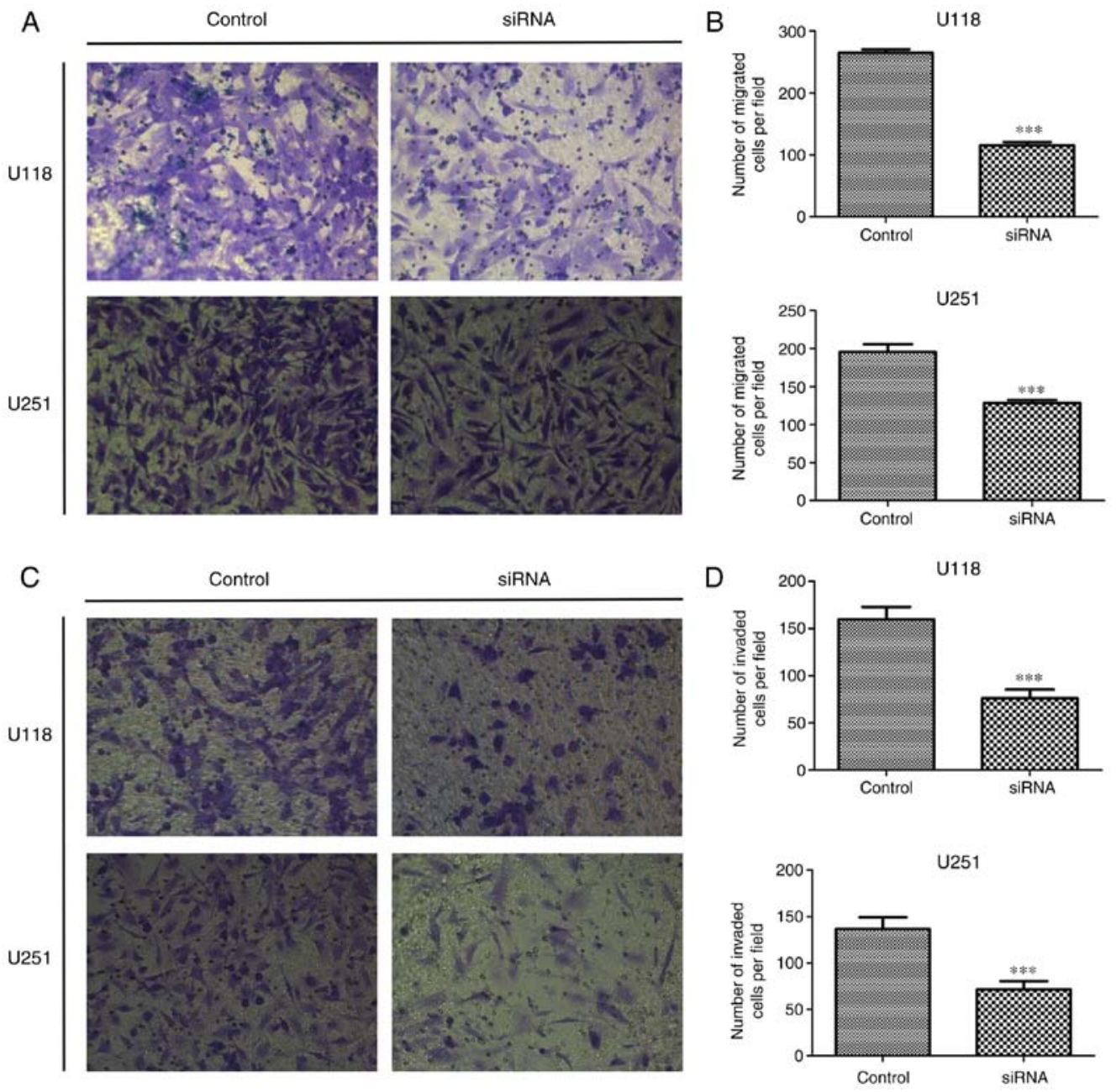

Figure 4. Knockdown of structure-specific recognition protein 1 (SSRP1) decreases cell migration and invasion. (A and B) Knockdown of SSRP1 decreases the migration ability of U118 and U251 cells. The representative images of migrated cells are shown (magnification, $\mathrm{x} 400$ ), and the migrated cells of each image are presented as the mean \pm standard deviation (SD). (C and D) Suppressed SSRP1 inhibited the invasion of U118 and U251 cells. Data are presented as the mean $\pm \mathrm{SD}$ of at least 3 independent experiments; ${ }^{* * *} \mathrm{P}<0.001$.

with scramble siRNA (negative control) ( $\mathrm{P}<0.05$; Fig. 2D). In addition, cell proliferation was also assessed using the EdU incorporation assays. As shown in Fig. 2E and F, the average percentage of EdU-positive cells in the control group and the SSRP1 knockdown group were 38.60 and $32.73 \%$ in the U118 cells, and 44.1 and $30.6 \%$ in the U251 cells $(\mathrm{P}<0.001)$. The knockdown of SSRP1 expression led to a marked decrease in the percentage of EdU-positive cells, as compared with the control group $(\mathrm{P}<0.001)$.

All the aforementioned results revealed that the downregulation of SSRP1 suppressed the proliferation of glioma cells in vitro.

siRNA-mediated knockdown of SSRPI arrests the cell cycle and causes apoptosis in glioma cells. In order to explore whether the effect of SSRP1 depletion on cell proliferation was related to the cell cycle and apoptosis, flow cytometric analysis was first performed. Cells transfected with si-SSRP1 exhibited a significant decrease in the percentage of cells in the $\mathrm{S}+\mathrm{G} 2 / \mathrm{M}$ phase compared with the cells transfected with scramble siRNA in the U118 (29.31 vs. $24.05 \%)$ and U251 cell lines (54.53 vs. $40.47 \%$ ) (Fig. 3A and B). Moreover, the function of SSRP1 in apoptosis was also investigated. After transfection with si-SSRP1 or scramble siRNA for $48 \mathrm{~h}$, the average percentage of apoptotic U118 cells treated with si-SSRP1 and the control group was 4.36 and $12.83 \%$, respectively ( $\mathrm{P}<0.001$; Fig. $3 \mathrm{C}$ and $\mathrm{D})$. Experiments using $\mathrm{U} 251$ yielded similar results, and the average percentage of apoptotic cells was significantly increased after treatment with si-SSRP1. The percentage of apoptotic U251 cells in the two groups was 8.18 and $21.83 \%$ ( $P<0.001$; Fig. $3 \mathrm{C}$ and D). These results revealed that SSRP1 was involved in the cell cycle process and apoptosis of glioma cells.

Decrease of SSRPI by siRNA inhibits glioma cell migration and invasion. In order to investigate the role of SSRP1 in glioma metastasis, we examined whether SSRP1 played a role in cell migration and invasion using the Transwell apparatus. After $24 \mathrm{~h}$ of incubation, the number of migrated cells in both the U118 and U251 cells transfected with si-SSRP1 was significantly lower than that in the negative control group $(\mathrm{P}<0.001$, for each; Fig. 4A and B). The Transwell matrix penetration assay was performed to evaluate the effects of SSRP1 on cell invasion. Compared with the control group, the cells with downregulated SSRP1 expression by siRNA demonstrated a significantly decreased invasion in both U118 and U251 cell lines $(\mathrm{P}<0.001$, for both; Fig. $4 \mathrm{C}$ and $\mathrm{D})$. All these results 

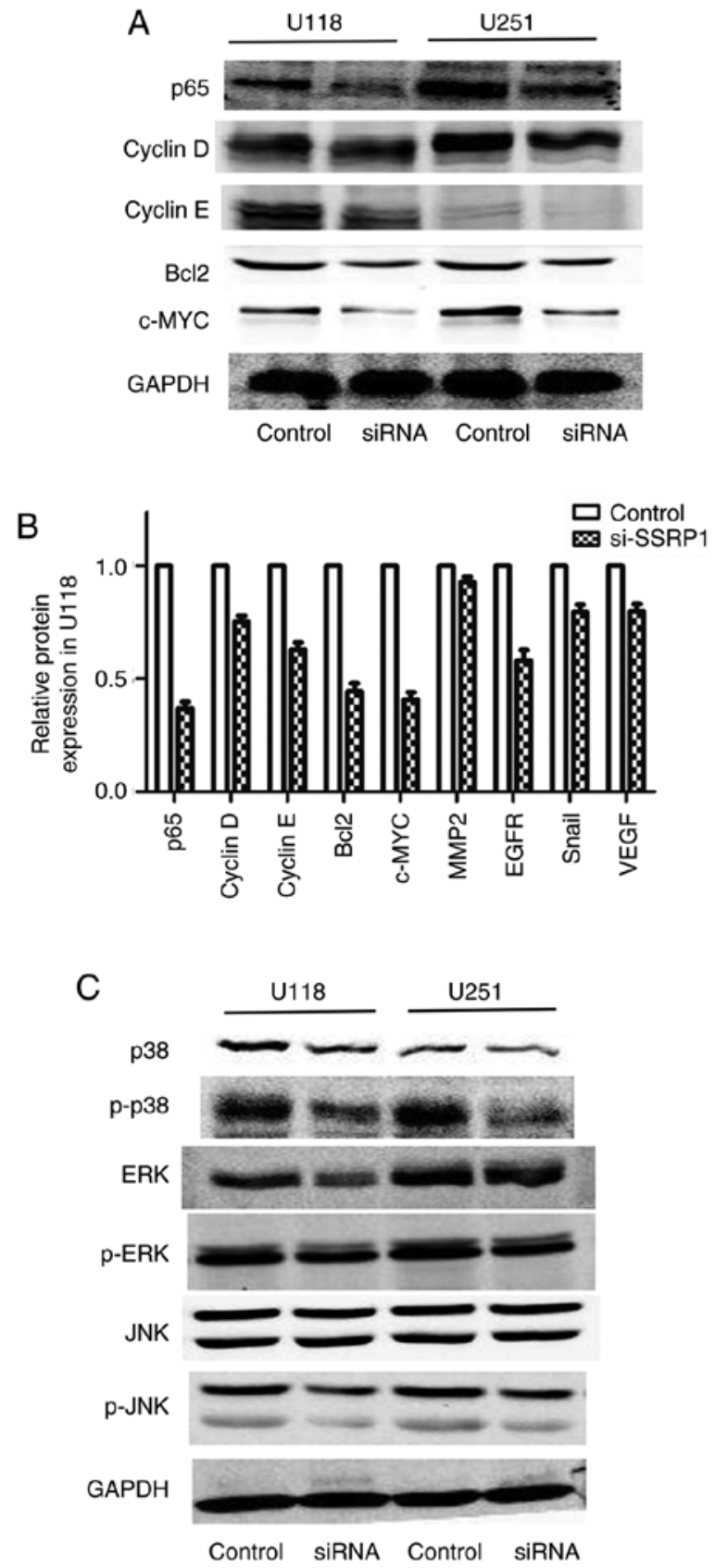
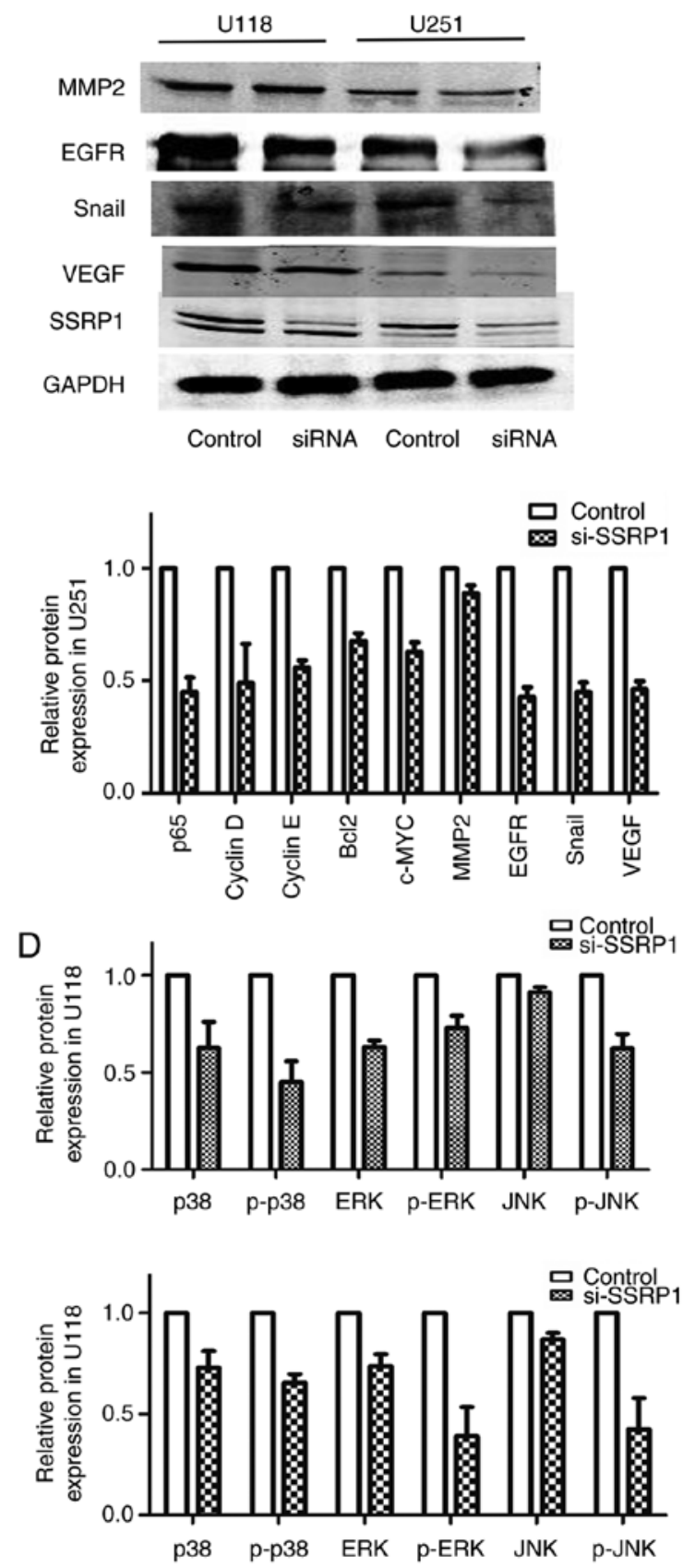

Figure 5. Structure-specific recognition protein 1 (SSRP1) regulates the expression of proliferation- and migration-associated genes in glioma via the MAPK pathway. (A and B) Knockdown of SSRP1 expression decreased the expression of proliferation-associated genes, including p65, c-myc, cyclin D and E. The expression of EGFR, VEGF and Snail, proteins involved in migration, were also suppressed. (C and D) The downregulated expression of SSRP1 significantly decreased the total and phosphorylated protein levels of p38 and ERK, and only the phosphorylated protein levels of JNK.

demonstrated that SSRP1 modulates glioma cell migration and invasion in vitro.

SSRP1 controls the expression of proliferation-and migration-associated genes in glioma. In order to explore the mechanism by which SSRP1 modulated the progression of cell proliferation and metastasis, the proteins involved in the cell cycle, migration and invasion in U118 and U251 cells were analyzed. The downregulated expression of SSRP1 by si-SSRP1 suppressed the protein levels of p65, c-myc and the cell cycle regulators including cyclin D and E (Fig. 5A). Compared to the negative control group, the protein levels of epithelial-mesenchymal transition and metastasis, including
EGFR, VEGF and Snail, decreased in the group transfected with si-SSRP1, but MMP2 did not decrease (Fig. 5A).

SSRP1 regulates the MAPK signaling pathway. The MAPK signaling pathway has an important role in regulating the proliferation and migration of glioblastoma cells. In order to evaluate the underlying mechanisms of SSRP1 in regulating glioma cell proliferation and migration, western blot assay was carried out to detect the alterations in the main proteins in the MAPK pathway. As shown in Fig. 5B, the downregulated expression of SSRP1 significantly decreased the phosphorylation of p38, ERK and JNK. Furthermore, the total p38 and ERK protein expression was also decreased in the group transfected 
with si-SSRP1, but not the total JNK protein level (Fig. 5B). These results revealed that SSRP1 is an upstream factor that modulated the MAPK signaling pathway in glioma.

\section{Discussion}

SSRP1 has been reported as a subunit of histone chaperone protein FACT, which causes the disruption of nucleosome and histone replacements $(21,22)$. It has been reported to be involved in the progression of DNA replication, DNA damage repair, cell proliferation and cell apoptosis $(14,15,23)$. Previous studies have focused on the molecular mechanism directing transcription elongation. However, few studies have reported the correlation between SSRP1 and cancer, and no study has focused on the clinical significance and oncogenicity effect of SSRP1 in glioma.

Our studies revealed that both the mRNA and protein levels of SSRP1 were upregulated in glioma tissues, compared with those in NB tissues, and that the SSRP1 protein level was higher in the high-grade glioma patients than in the low-grade glioma patients, which were identical to the results of the GEO datasets analyses. These findings suggest that SSRP1 may play an important role in the development of human glioma, and that the overexpression level may predict highgrade glioma.

The overexpression of SSRP1 in glioblastoma predicted the oncogenic role of SSRP1 in glioma tumorigenesis. First, we used multiple methods, including the CCK-8 and EdU incorporation assays, and FACS flow cytometric analysis, to identify the role of SSRP1 in GBM cell growth progression. Furthermore, we demonstrated that the viability and proliferation abilities were significantly decreased in glioma cells with the silencing of SSRP1 by siRNA transfection. In addition, we also ascertained the biological function of SSRP1 on GBM cell motility, and detected that the knockdown of SSRP1 by siRNA inhibited glioma cell migration and invasion. Glioblastoma is the most common aggressive phenotype of glioma, and is identified by the characteristics of cellular proliferation, diffuse infiltration, necrosis, angiogenesis and intense resistance to apoptosis (24). The results of the present study reflected that the decreased expression of SSRP1 inhibited the ability of cell proliferation, anti-apoptosis and metastasis, which were the hallmark features of glioblastoma.

In order to further confirm the function of SSRP1, we examined some cell growth regulators and metastasis markers. It was observed that the protein levels of proliferation-associated genes, including p65, c-myc, cyclin D and E, were inhibited after SSRP1 downregulation in glioma U118 and U251 cells. Furthermore, we demonstrated that SSRP1 knockdown suppressed the expression of metastasis-associated genes such as EGFR, VEGF and Snail.

The MAPK pathway regulated multiple cellular programs including embryogenesis, proliferation, apoptosis and differentiation, based on cues derived from the surface and metabolic state of the cells (25). The 3 major MAPK pathways are ERKs, JNKs and the p38 families (26). In the present study, we also demonstrated that the downregulated expression of SSRP1 significantly decreased the phosphorylation of p38, ERK and JNK, and that total p38 and ERK protein expression was also decreased, but not the total JNK protein level. Hence, the present study revealed that SSRP1 may be involved in tumor progression via the MAPK pathway.

In conclusion, the present study indicated that SSRP1 may regulate the function of the MAPK pathway to promote cell proliferation and metastasis in gliomas. SSRP1 overexpression in glioma specimens could be used for the diagnosis of glioma, and our results revealed the potential role for SSRP1 in glioma therapy. In addition, further research to clarify the molecular mechanism of SSRP1 involved in glioma tumorigenesis is warranted.

\section{Acknowledgements}

The present study was supported by grants from the National Science Foundation of China (nos. 81502175 and 81572489).

\section{References}

1. Chen W, Zheng R, Baade PD, Zhang S, Zeng H, Bray F, Jemal A, Yu XQ and He J: Cancer statistics in China, 2015. CA Cancer J Clin 66: 115-132, 2016.

2. Ohgaki $\mathrm{H}$ and Kleihues $\mathrm{P}$ : Genetic pathways to primary and secondary glioblastoma. Am J Pathol 170: 1445-1453, 2007.

3. Baumert BG, Hegi ME, van den Bent MJ, von Deimling A, Gorlia T, Hoang-Xuan K, Brandes AA, Kantor G, Taphoorn MJ, Hassel MB, et al: Temozolomide chemotherapy versus radiotherapy in high-risk low-grade glioma (EORTC 22033-26033): A randomised, open-label, phase 3 intergroup study. Lancet Oncol 17: 1521-1532, 2016.

4. Norden AD, Drappatz J and Wen PY: Novel anti-angiogenic therapies for malignant gliomas. Lancet Neurol 7: 1152-1160, 2008 .

5. Belotserkovskaya R, Oh S, Bondarenko VA, Orphanides G, Studitsky VM and Reinberg D: FACT facilitates transcriptiondependent nucleosome alteration. Science 301: 1090-1093, 2003.

6. Röttgers K, Krohn NM, Lichota J, Stemmer C, Merkle T and Grasser KD: DNA-interactions and nuclear localisation of the chromosomal HMG domain protein SSRP1 from maize. Plant J 23: 395-405, 2000

7. Birch JL, Tan BC, Panov KI, Panova TB, Andersen JS, Owen-Hughes TA, Russell J, Lee SC and Zomerdijk JC: FACT facilitates chromatin transcription by RNA polymerases I and III. EMBO J 28: 854-865, 2009.

8. Kumari A, Mazina OM, Shinde U, Mazin AV and Lu H: A role for SSRP1 in recombination-mediated DNA damage response. J Cell Biochem 108: 508-518, 2009.

9. Mason PB and Struhl K: The FACT complex travels with elongating RNA polymerase II and is important for the fidelity of transcriptional initiation in vivo. Mol Cell Biol 23: 8323-8333, 2003.

10. Tan BC, Liu H, Lin CL and Lee SC: Functional cooperation between FACT and MCM is coordinated with cell cycle and differential complex formation. J Biomed Sci 17: 11, 2010.

11. Zhang W, Zeng F, Liu Y, Shao C, Li S, Lv H, Shi Y, Niu L, Teng $M$ and Li X: Crystal structure of human SSRP1 middle domain reveals a role in DNA binding. Sci Rep 5: 18688, 2015.

12. Garcia H, Fleyshman D, Kolesnikova K, Safina A, Commane M, Paszkiewicz G, Omelian A, Morrison C and Gurova K: Expression of FACT in mammalian tissues suggests its role in maintaining of undifferentiated state of cells. Oncotarget 2 : 783-796, 2011.

13. Garcia H, Miecznikowski JC, Safina A, Commane M, Ruusulehto A, Kilpinen S, Leach RW, Attwood K, Li Y, Degan S, et al: Facilitates chromatin transcription complex is an 'accelerator' of tumor transformation and potential marker and target of aggressive cancers. Cell Reports 4: 159-173, 2013.

14. Ding Q, He K, Luo T, Deng Y, Wang H, Liu H, Zhang J, Chen K, Xiao J, Duan X, et al: SSRP1 contributes to the malignancy of hepatocellular carcinoma and is negatively regulated by miR-497. Mol Ther 24: 903-914, 2016.

15. Dermawan JK, Gurova K, Pink J, Dowlati A, De S, Narla G, Sharma N and Stark GR: Quinacrine overcomes resistance to erlotinib by inhibiting FACT, NF- $\kappa \mathrm{B}$, and cell-cycle progression in non-small cell lung cancer. Mol Cancer Ther 13: 2203-2214, 2014. 
16. Koman IE, Commane M, Paszkiewicz G, Hoonjan B, Pal S, Safina A, Toshkov I, Purmal AA, Wang D, Liu S, et al: Targeting FACT complex suppresses mammary tumorigenesis in Her2/neu transgenic mice. Cancer Prev Res 5: 1025-1035, 2012.

17. Carter DR, Murray J, Cheung BB, Gamble L, Koach J, Tsang J, Sutton S, Kalla H, Syed S, Gifford AJ, et al: Therapeutic targeting of the MYC signal by inhibition of histone chaperone FACT in neuroblastoma. Sci Transl Med 7: 312ra176, 2015.

18. Lake D, Corrêa SA and Müller J: Negative feedback regulation of the ERK1/2 MAPK pathway. Cell Mol Life Sci 73: 4397-4413, 2016.

19. Wang Z, Guo Q, Wang R, Xu G, Li P, Sun Y, She X, Liu Q, Chen Q, Yu Z, et al: The D Domain of LRRC4 anchors ERK1/2 in the cytoplasm and competitively inhibits MEK/ERK activation in glioma cells. J Hematol Oncol 9: 130, 2016.

20. Shingu T, Holmes L, Henry V, Wang Q, Latha K, Gururaj AE, Gibson LA, Doucette T, Lang FF, Rao G, et al: Suppression of $\mathrm{RAF} / \mathrm{MEK}$ or PI3K synergizes cytotoxicity of receptor tyrosine kinase inhibitors in glioma tumor-initiating cells. J Transl Med 14: 46, 2016.
21. Takahata S, Yu Y and Stillman DJ: FACT and Asf1 regulate nucleosome dynamics and coactivator binding at the $\mathrm{HO}$ promoter. Mol Cell 34: 405-415, 2009.

22. Tsunaka Y, Fujiwara Y, Oyama T, Hirose S and Morikawa K: Integrated molecular mechanism directing nucleosome reorganization by human FACT. Genes Dev 30: 673-686, 2016.

23. Gao XJ, Feng JX, Zhu S, Liu XH, Tardieux I and Liu LX: Protein phosphatase $2 \mathrm{C}$ of toxoplasma gondii interacts with human SSRP1 and negatively regulates cell apoptosis. Biomed Environ Sci 27: 883-893, 2014.

24. Furnari FB, Fenton T, Bachoo RM, Mukasa A, Stommel JM, Stegh A, Hahn WC, Ligon KL, Louis DN, Brennan C, et al: Malignant astrocytic glioma: Genetics, biology, and paths to treatment. Genes Dev 21: 2683-2710, 2007.

25. Raman M, Chen W and Cobb MH: Differential regulation and properties of MAPKs. Oncogene 26: 3100-3112, 2007.

26. Owens DM and Keyse SM: Differential regulation of MAP kinase signalling by dual-specificity protein phosphatases. Oncogene 26: 3203-3213, 2007. 